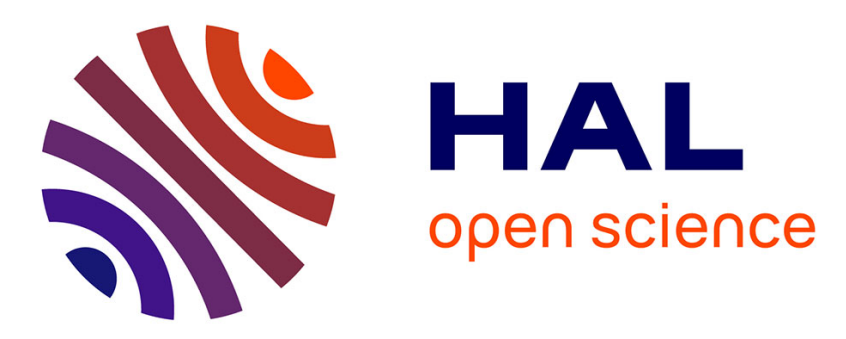

\title{
A Fast Adaptive Method for Subspace Based Blind Channel Estimation
}

\author{
Jon Altuna, Bernie Mulgrew, Roland Badeau, Vicente Atxa
}

\section{To cite this version:}

Jon Altuna, Bernie Mulgrew, Roland Badeau, Vicente Atxa. A Fast Adaptive Method for Subspace Based Blind Channel Estimation. Proc. of IEEE International Conference on Acoustics, Speech and Signal Processing (ICASSP), 2006, Toulouse, France. pp.1121-1124. hal-00945268

\section{HAL Id: hal-00945268 \\ https://hal.inria.fr/hal-00945268}

Submitted on 24 Mar 2014

HAL is a multi-disciplinary open access archive for the deposit and dissemination of scientific research documents, whether they are published or not. The documents may come from teaching and research institutions in France or abroad, or from public or private research centers.
L'archive ouverte pluridisciplinaire HAL, est destinée au dépôt et à la diffusion de documents scientifiques de niveau recherche, publiés ou non, émanant des établissements d'enseignement et de recherche français ou étrangers, des laboratoires publics ou privés. 


\title{
A FAST ADAPTIVE METHOD FOR SUBSPACE BASED BLIND CHANNEL ESTIMATION
}

\author{
A. Jon Altuna, B. Bernie Mulgrew*, C. Roland Badeau ${ }^{\dagger}$ and D. Vicente Atxa \\ University of Mondragón \\ Department of Electronics \\ 20500 Mondragon, Spain
}

\begin{abstract}
In this paper, a new fast adaptive blind channel estimation method is proposed using the subspace information from the correlation matrix. The algorithm is fully adaptive in the sense that both the subspace information and the optimization which leads to the channel estimation are computed adaptively. It is based on the recently proposed YAST subspace tracker which has been shown to outperform other methods both in terms of speed of convergence and computational complexity. A discussion on the convergence properties of the proposed algorithm is presented. We also propose a hybrid method which makes use of the YAST subspace tracker for initial fast convergence and the subspace information is then updated using the numerically stable OPAST subspace tracker.
\end{abstract}

\section{INTRODUCTION}

The increasing demand for high spectral efficiency in broadband wireless communications where multipath delay spread is a major problem has motivated the design of (semi-) blind channel estimation and equalization techniques which require little or no knowledge of the transmitted sequence. A number of (semi-) blind techniques have been proposed, which rely on some statistical properties or algebraic structure of the received signal [2]. In particular, several subspace techniques have been proposed for blind and semi-blind channel identification after the pioneering work by Moulines et al. [1] which has been applied to single-carrier [5] and multicarrier systems [3]. These algorithms generally require the computation of an SVD on the received signal correlation matrix to determine the signal and noise subspace in a first step and then the channel impulse response is estimated as the solution to a quadratic form up to a scalar factor.

Recent developments in subspace tracking have shown that performance matching a detailed SVD can be obtained using subspace projection based algorithms [7] [6]. These algorithms view the estimation of the signal subspace as an optimization problem and compute the signal subspace as the exact solution over a subspace of reduced dimensions. Essentially, an orthonormal matrix which spans the $n$-dimensional dominant subspace of the correlation matrix is adaptively estimated where the orthonormality of the estimated projection matrix is guaranteed at each iteration. In particular, the YAST algorithm proposed in [6] clearly outperforms subspace trackers such as OPAST [9] or plane-rotation based FST [8] with a global complexity $O(m n)$ where $m$ is the dimension of

${ }^{*}$ The author is with the Institute for Digital Communications (IDCOM), University of Edinburgh

${ }^{\dagger}$ The author is with the Department of Signal and Image Processing, Télécom Paris the observed data window. Recently, a promising subspace tracker known as FAPI [10] has also been presented, which outperforms OPAST both in terms of stability and speed of convergence, but YAST has a much faster initial convergence than FAPI.

This paper presents a fast fully-adaptive subspace-based blind channel estimation method. We first propose a method which uses the YAST subspace tracker to adaptively calculate the signal subspace from the correlation matrix and the quadratic form solution is also adaptively calculated using the iterative power method [4]. However, we have observed that, although the YAST algorithm converges remarkably at a similar speed of a detailed SVD with an important reduction in computational complexity, the algorithm diverges when the iterations reach the dimension of the signal subspace. Nevertheless, we propose a hybrid approach which combines the YAST subspace tracker for initial fast convergence and stability is guaranteed by updating the subspace information using the numerically stable OPAST subspace tracker. The proposed method is numerically stable and achieves faster convergence than other methods proposed in the literature [4] with a global complexity of $O(m n)$.

The paper is organized as follows: in Section 2 the multichannel system model is presented and Section 3 presents the fully adaptive blind channel estimation method based on the YAST subspace tracker. Section 4 discusses convergence properties of the adaptive algorithm and proposes a hybrid method which guarantees stability. Finally, computer simulations are presented in Section 5 which highlight the ability of the proposed hybrid approach to track the impulse response of a frequency-selective propagation channel faster than other methods using different subspace trackers.

\section{SYSTEM MODEL}

The system is described as a multichannel system where the discrete time input sequence $x(k)$ is received by $K$ antennas or equivalently is sampled at a rate $K$-times faster than the source symbol rate. The received signal $y^{(i)}(k)$ at the $i$ th subchannel is formulated as:

$$
\mathbf{y}_{k}^{(i)}=\mathbf{H}_{N}^{(i)} \mathbf{x}_{k}+\mathbf{w}_{k}^{(i)}
$$

where $\mathbf{x}_{k}=[x(k), x(k-1), \cdots, x(k-N-M+1)]^{\dagger}$ is the $(N+M) \times 1$ vector which contains the input sequence $x(k)$, $N$ represents the dimension of the data window at each subchannel and $M+1$ is the length of the subchannel impulse response. On the other hand, $\mathbf{y}_{k}^{(i)}=\left[y^{(i)}(k), y^{(i)}(k-1), \cdots, y^{(i)}(k-\right.$ $N+1)]^{\dagger}$ represents the $N \times 1$ received signal vector and $\mathbf{w}_{k}^{(i)}=$ 
$\left[w^{(i)}(k), w^{(i)}(k-1), \cdots, w^{(i)}(k-N+1)\right]^{\dagger}$ is the noise vector. The subchannel filtering matrix $\mathbf{H}_{N}^{(i)}$ is a $N \times(N+M)$ Block-Toeplitz matrix associated with the $i$ th subchannel impulse response $h_{k}^{(i)} \stackrel{\text { def }}{=} h^{(i)}(k)$ as:

$$
\mathbf{H}_{N}^{(i)}=\left(\begin{array}{ccccccc}
h_{0}^{(i)} & \ldots & h_{M}^{(i)} & 0 & \ldots & \ldots & 0 \\
0 & h_{0}^{(i)} & \cdots & h_{M}^{(i)} & 0 & \ldots & 0 \\
\vdots & \vdots & \vdots & \vdots & \vdots & \vdots & \vdots \\
0 & \ldots & \ldots & 0 & h_{0}^{(i)} & \ldots & h_{M}^{(i)}
\end{array}\right)
$$

Equivalently,

$$
\mathbf{y}_{k}=\mathbf{H}_{N} \mathbf{x}_{k}+\mathbf{w}_{k}
$$

where $\mathbf{y}_{k} \stackrel{\text { def }}{=}\left[\mathbf{y}_{k}^{(0) \dagger}, \cdots, \mathbf{y}_{k}^{(K-1) \dagger}\right]^{\dagger}$ is the received signal vector and $\mathbf{H}_{N}=\left[\mathbf{H}_{N}^{(0) \dagger}, \cdots, \mathbf{H}_{N}^{(K-1) \dagger}\right]^{\dagger}$ is a $K N \times(N+M)$ filtering matrix.

\section{ADAPTIVE SUBSPACE-BASED BLIND CHANNEL ESTIMATION}

The identification of the channel coefficients is based on the SVD of the $K N \times K N$ covariance matrix $\mathbf{R}_{y}(k)$ of the received signal. Provided that the filtering matrix $\mathbf{H}_{N}$ meets the full column-rank condition, the observation window $N$ is sufficiently large so that $N \geq M$ and the subschannels $h_{k}^{(i)}$ do not share common zeros [1], the singular vectors $\mathbf{S}(k)$ associated with the $M+N$ highest singular values span the signal subspace and the singular vectors $\mathbf{G}(k)$ associated with the last $K N-M-N$ singular values span the noise-subspace:

$$
\begin{aligned}
& \mathbf{R}_{y}(k)=\mathbf{H}_{N} \mathbf{R}_{x}(k) \mathbf{H}_{N}^{H}+\mathbf{R}_{w}(k) \\
& =[\mathbf{S}(k) \mathbf{G}(k)]\left[\begin{array}{ll}
\boldsymbol{\Sigma}_{S}(k) & \mathbf{0} \\
\mathbf{0} & \mathbf{\Sigma}_{G}(k)
\end{array}\right]\left[\begin{array}{l}
\mathbf{S}(k)^{H} \\
\mathbf{G}(k)^{H}
\end{array}\right]
\end{aligned}
$$

where $\mathbf{R}_{w}(k)$ is the autocorrelation matrix of the stationary noise process.

Since the signal subspace is a linear space spanned by the filtering matrix $\mathbf{H}_{N}$, according to [1], the subschannel impulse responses $h_{k}^{(i)}$ can be estimated by maximizing the following quadratic form:

$$
\begin{aligned}
q^{\prime}(H) & =\sum_{i=0}^{M+N-1}\left|\hat{\mathbf{S}}_{i}(k)^{H} \mathbf{H}_{N}\right|^{2} \\
& =H^{H}\left(\sum_{i=0}^{M+N-1} \hat{S}_{i}(k) \hat{S}_{i}(k)^{H}\right) H \\
& =H^{H} \hat{Q}(k) H
\end{aligned}
$$

$H=\left[H^{(0) T}, \ldots, H^{(K-1) T}\right]$ is a $1 \times K(M+1)$ channel vector and $H^{(i)}=\left[h_{0}^{(i)}, \ldots, h_{M}^{(i)}\right]^{T} . \hat{\mathbf{S}}_{i}(k)$ are the estimated signal subspace singular vectors and $\hat{S}_{i}(k)$ is a transformation matrix such that:

$$
\hat{S}_{i}(k)=\Upsilon_{M+1}\left[\hat{\mathbf{S}}_{i}(k)\right],
$$

where $\mathbf{H}_{N}^{(i)}=\Upsilon_{N}\left[H^{(i)}\right]$.

The solution to this maximization problem is the unit-norm singular vector associated with the maximum singular value of matrix $\hat{Q}(k)$ subject to the unit norm constraint $\|H\|=1$.
In particular, we propose an adaptive method for the estimation of the signal subspace based on maximizing the following criterion:

$$
\tau[\hat{\mathbf{S}}(k)]=\operatorname{trace}\left[\hat{\mathbf{S}}(k){ }^{H} \hat{\mathbf{R}}_{y}(k) \hat{\mathbf{S}}(k)\right]
$$

where $\hat{\mathbf{S}}(k)$ is the estimated $m \times n$ orthonormal matrix which spans the signal subspace and $m=K N$ and $n=M+N$. This maximization is subject to the recursive estimation of the received signal covariance matrix as:

$$
\hat{\mathbf{R}}_{y}(k)=\beta \hat{\mathbf{R}}_{y}(k-1)+\mathbf{y}_{k} \mathbf{y}_{k}^{H}
$$

The idea introduced in [7] and [6] is to reduce the search space of the $m \times n$ orthonormal vectors $\hat{\mathbf{S}}(k)$ which requires an overall complexity $O\left(m^{2} n\right)$ by maximizing the following alternative criterium instead:

$$
\begin{aligned}
\tau[\hat{\mathbf{S}}(k)] & =\operatorname{trace}\left[\mathbf{R}_{z}(k)\right] \\
& =\operatorname{trace}[U(k)^{H} \underbrace{\tilde{\mathbf{S}}(k)^{H} \mathbf{R}_{y}(k) \tilde{\mathbf{S}}(k)} U(k)] \\
& =\operatorname{trace}\left[U(k)^{H} \tilde{\mathbf{R}}_{z}(k) U(k)\right]
\end{aligned}
$$

where $\hat{\mathbf{S}}(k)=\tilde{\mathbf{S}}(k) U(k)$. The matrix $\tilde{\mathbf{S}}(k)=[\hat{\mathbf{S}}(k-1), V(k)]$ is an orthogonal matrix with $p=1$ or $p=2$ more columns appended to matrix $\hat{\mathbf{S}}(k-1)$. On the other hand, $V(k)=\mathbf{e}_{k} / \operatorname{norm}\left(\mathbf{e}_{k}\right)$ is the normalized prediction error, where:

$$
\mathbf{e}_{k}=\mathbf{x}_{k}-\hat{\mathbf{S}}(k-1) \hat{\mathbf{S}}(k-1)^{H} \mathbf{x}_{k}
$$

The maximization of eq. 9 reduces the search space from $m \times$ $n$ to the $(n+p) \times n$ orthonormal matrix $U(k)$ which spans the $n$-dimensional dominant subspace of $\tilde{\mathbf{R}}_{z}(k)$, but it would still require to compute an singular value decomposition with a complexity $O\left(n^{3}\right)$. In order to avoid this, we take into account the fact that the orthonormal matrix $U(k)$ also spans an invariant subspace of the inverse $\tilde{\mathbf{R}}_{z}(k)^{-1}$

$$
\mathbf{R}_{z}(k)^{-1}=U(k)^{H} \tilde{\mathbf{R}}_{z}(k)^{-1} U(k)
$$

Thus, the YAST algorithm computes $\tilde{\mathbf{R}}_{z}(k)^{-1}$ and its $p$ - dimensional dominant subspace which does not require any singular value decomposition. This optimization leads to a simple recursion for the adaptation rule of the signal subspace matrix [6]:

$$
\begin{aligned}
\hat{\mathbf{S}}(k)= & \hat{\mathbf{S}}(k-1)-\tilde{\zeta}(k) \tilde{\mathbf{f}}_{k}^{H}=\hat{\mathbf{S}}(k-1) \\
& -\left[V(k)+\hat{\mathbf{S}}(k-1) \tilde{\mathbf{f}}_{k}(\tilde{\mathbf{I}}+\tilde{\rho}(k))^{-1}\right] \tilde{\mathbf{f}}_{k}^{H}
\end{aligned}
$$

where $\tilde{\mathbf{f}}_{k}=\tilde{\varphi}(k) \tilde{\theta}(k)^{H}$. For the sake of simplicity we study the adaptation rule for $p=1$ rule. Then, $\tilde{\varphi}(k)$ is built from the first $m$ coefficients of the $(m+1) \times 1$ dominant singular vector of the matrix $\tilde{\mathbf{R}}_{z}(k)^{-1}$, and $\tilde{\theta}(k)$ and $\tilde{\rho}(k)$ correspond to the sign and the absolute value of the last coefficient of this singular vector. The adaptation of the signal subspace matrix $\hat{\mathbf{S}}(k)$ requires $4 m n+$ $O\left(m+n^{2}\right)$ MAC (multiply-accumulate) operations.

On the other hand, the solution to the maximization of the quadratic form expressed in eq. 5 would require the computation of the dominant singular vector of the $m_{1} \times m_{1}$ matrix $\hat{Q}(k)$ with a complexity $O\left(m_{1}^{2}\right)$, where $m_{1}=K(M+1)$. In this case, a further reduction in complexity can be achieved by tracking the most significant singular vector of matrix $\hat{Q}(k)$ using the power method [11]. The power method is an iterative method for computing the dominant singular vector with a complexity in each iteration. The procedure is as follows: 
Since the matrix $\hat{Q}(k)$ is a symmetric positive matrix, the dominant left singular vector $U_{q}(k)$ of $\hat{Q}(k)$ can be directly obtained according to:

$$
\begin{aligned}
U_{q}(k) & =\hat{Q}(k) U_{q}(k-1) \\
U_{q}(k) & =U_{q}(k) /\left\|U_{q}(k)\right\|
\end{aligned}
$$

Finally, the channel vector estimate can be obtained from the dominant left singular vector $U_{q}(k)=\left[u_{q}^{(0)}, \cdots, u_{q}^{(K(M+1))}\right]$ us$\operatorname{ing} H^{(i)}=\left[u_{q}^{(i)}, u_{q}^{(K+i)}, \cdots, u_{q}^{(K M+i)}\right]$.

In summary, the adaptive blind channel estimation algorithm would require the following steps:

Table I

Adaptive Blind Channel Estimation

\begin{tabular}{ll}
\hline \hline Recursion: \\
\hline for $\quad k=1,2, \ldots$ \\
& Compute $\hat{\mathbf{R}}_{y}(k)=\beta \hat{\mathbf{R}}_{y}(k-1)+\mathbf{y}_{k} \mathbf{y}_{k}^{H}$ \\
& Update $\hat{\mathbf{S}}(k)=\hat{\mathbf{S}}(k-1)-\tilde{\zeta}(k) \tilde{\mathbf{f}}_{k}^{H}($ eq. 12) \\
& Compute $\hat{Q}(k)=\left(\sum_{i=0}^{M+N-1} \hat{S}_{i}(k) \hat{S}_{i}(k)^{H}\right)$ \\
& Update $U_{q}(k)$ using $U_{q}(k)=\hat{Q}(k) U_{q}(k-1)$ \\
& and $U_{q}(k)=U_{q}(k) /\left\|U_{q}(k)\right\|$ \\
end & \\
\hline \hline
\end{tabular}

\section{CONVERGENCE PROPERTIES AND HYBRID METHOD}

In this section we consider the convergence properties of the YAST subspace tracker in the context of blind channel estimation. In particular, it is interesting to note that after $k=M+N$ iterations, the signal subspace is somewhat estimated as the $M+N$-dimensional subspace of $\left[\hat{\mathbf{S}}(k-1), \mathbf{x}_{k}, \mathbf{x}_{k+1}, \ldots, \mathbf{x}_{k+M+N-1}\right]$ which maximizes the Rayleigh quotient. However, the family $\mathbf{x}_{k}, \mathbf{x}_{k+1}, \ldots$, $\mathbf{x}_{k+M+N-1}$ exactly spans the true signal subspace and therefore, after $k=M+N$ iterations the estimated subspace matches the true signal subspace. This explanation only holds for noiseless signals.

On the other hand, we have observed that the YAST algorithm diverges immediately after convergence. This is caused by the loss of orthonormality of the updated matrix $\hat{\mathbf{S}}(k)$ due to the fact that the prediction error $\mathbf{e}_{k}=\mathbf{x}_{k}-\hat{\mathbf{S}}(k-1) \hat{\mathbf{S}}(k-1)^{H} \mathbf{x}_{k}$ becomes neglegible at iteration $k=M+N$. Indeed, when the algorithm reaches convergence at $k=M+N$ we obtain an orthonormal matrix $\hat{\mathbf{S}}(k)$ which matches the exact SVD solution for the same iteration. In practice, it is observed that the normalized prediction error vector $V(k)=\mathbf{e}_{k} / \operatorname{norm}\left(\mathbf{e}_{k}\right)$ in $\tilde{\mathbf{S}}(k)=[\hat{\mathbf{S}}(k-1), V(k)]$ is not exactly orthogonal to $\hat{\mathbf{S}}(k-1)$.

In most cases, especially when the observation window $N$ is small, the channel estimates are not able to converge to a reasonable value and we thus propose to use the YAST subspace tracker as an initialization with fast convergence for $k=M+N$ iterations and after that, the numerically stable OPAST subspace tracker [9] can be used for the adaptation of the signal subspace matrix.

The recursion used by the OPAST algorithm for the adaptation of the signal subspace is:

$$
\hat{\mathbf{S}}(k)=\hat{\mathbf{S}}(k-1)+\tilde{\mathbf{p}}_{k} \mathbf{q}_{k}^{H}
$$

where $\tilde{\mathbf{p}}_{k}=\tau_{k} \hat{\mathbf{S}}(k-1) \mathbf{q}_{k}+\left(1+\tau_{k}\left\|\mathbf{q}_{k}\right\|^{2}\right) \mathbf{p}_{k}$. On the other hand, we define the following terms as:

$$
\begin{gathered}
\mathbf{p}_{k}=\gamma_{k} \mathbf{e}_{k}, \\
\gamma_{k}=1 /\left(1+\mathbf{x}_{k}^{H} \hat{\mathbf{S}}(k-1) \mathbf{q}_{k}\right), \\
\mathbf{q}_{k}=1 / \beta\left(\mathbf{Z}(k-1) \hat{\mathbf{S}}(k-1)^{H} \mathbf{x}_{k}\right) \\
\mathbf{Z}(k)=(1 / \beta) \mathbf{Z}(k-1)-\gamma_{k} \mathbf{q}_{k} \mathbf{q}_{k}^{H}
\end{gathered}
$$

and

$$
\tau_{k}=\frac{1}{\left\|\mathbf{q}_{k}\right\|^{2}}\left(\frac{1}{\sqrt{1+\left\|\mathbf{p}_{k}\right\|^{2}\left\|\mathbf{q}_{k}\right\|^{2}}}-1\right) .
$$

This adaptation rule requires $4 m n+O\left(n^{2}\right)$ MAC (multiply-accumulate) operations. The pseudo-code of the proposed hybrid method is as follows

Table II

Hybrid Method

\begin{tabular}{ll}
\hline \hline Recursion: \\
\hline for $\quad k=1,2, \ldots$ \\
& Compute $\mathbf{R}_{y}(k)=\beta \mathbf{R}_{y}(k-1)+\mathbf{y}_{k} \mathbf{y}_{k}^{H}$ \\
& if $k \leq M+N$ \\
& $\quad$ Update $\hat{\mathbf{S}}(k)$ according to eq. 12 \\
& else \\
& Update $\hat{\mathbf{S}}(k)$ according to eq. 14 \\
& Compute $\hat{Q}(k)=\left(\sum_{i=0}^{M+N-1} \hat{S}_{i}(k) \hat{S}_{i}(k)^{H}\right)$ \\
& Update $U_{q}(k)$ using $U_{q}(k)=\hat{Q}(k) U_{q}(k-1)$ \\
& and $U_{q}(k)=U_{q}(k) /\left\|U_{q}(k)\right\|$ \\
end & \\
\hline \hline
\end{tabular}

The computational complexity of the different adaptive subspace based channel estimation techniques is summarized in Table III:

Table III

\begin{tabular}{|l|l|l|}
\hline \hline & $\begin{array}{l}\text { subspace } \\
\text { estimation }\end{array}$ & $\begin{array}{l}\text { estimation of } \\
U_{q}(k)\end{array}$ \\
\hline Detailed SVD & $O\left[(K N)^{3}\right]$ & {$[K(M+1)]^{2}$} \\
\hline YAST & $\begin{array}{l}4 K N(M+N) \\
+O\left[K N+(M+N)^{2}\right]\end{array}$ & {$[K(M+1)]^{2}$} \\
\hline OPAST & $\begin{array}{l}4 K N(M+N) \\
+O\left[(M+N)^{2}\right]\end{array}$ & {$[K(M+1)]^{2}$} \\
& YAST/OPAST & {$[K(M+1)]^{2}$} \\
\hline Hybrid & \multicolumn{2}{|l}{} \\
\hline \hline
\end{tabular}

\section{SIMULATION RESULTS}

Computer simulations have been conducted in order to compare the performance of the proposed fully adaptive method with the blind subspace technique using exact SVD and an adaptive method using the OPAST subspace tracker. The source signal is a $2 \mathrm{Mbps}$ BPSK signal which is received by $K=4$ antennas. The performance of these techniques is measured in terms of the normalized mean squared error (NMSE) of the estimated propagation subchannel impulse responses. The experiments were conducted using a frequency- selective SUI-3 channel model with [0 0.5 1] tap delay in $\mu \mathrm{s},[0-5-10]$ power in each tap in $\mathrm{dB}$ and $M=2$. The maximum Doppler frequency is $0.4 \mathrm{~Hz}$. 


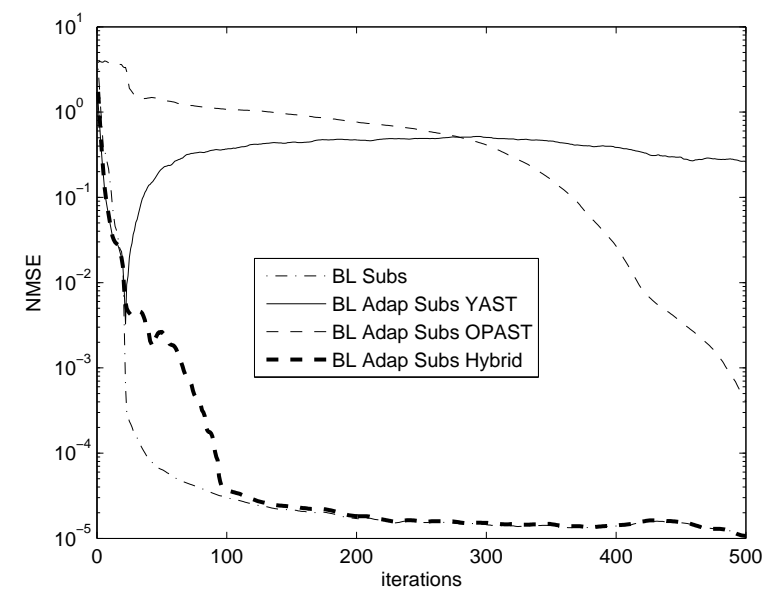

Fig. 1. Convergence of channel estimation using an observation window size $N=20$ and $\mathrm{SNR}=25 \mathrm{~dB}$.

Figure 1 shows the convergence of the NMSE averaged over 50 Monte-Carlo runs, using a fixed observation window size $N=$ 20 and $\mathrm{SNR}=25 \mathrm{~dB}$. It is observed that the adaptive method using the YAST algorithm the signal subspace estimation and the iterative power method for the quadratic form solution is able to converge to the solution provided by the exact SVD-based method (denoted BL Subs). However, it can be seen that the algorithm diverges immediately after convergence at $k=M+N$. On the other hand, we have also tested the performance of the adaptive technique using the OPAST subspace tracker and it is apparent that the convergence is slow compared to the YAST subspace tracker. We finally tested the proposed hybrid method which switches from the YAST adaptation rule to OPAST at $k=M+N$ and it is shown that this combination enables global convergence of the algorithm while increasing the speed of convergence.

Another experiment was also conducted computing the NMSE over 100 Monte-Carlo runs using a fixed window size $N=20$. It is observed in Figure 2 that the proposed hybrid adaptive technique achieves almost identical performance as the exact SVDbased technique at iterations $k=22$ and $k=150$, whereas YAST diverges after iteration $k=22$ and the OPAST based adaptive method is not even able to converge at $k=150$.

\section{CONCLUSIONS}

In this paper we propose a new method for adaptive subspacebased blind channel estimation using the YAST subspace tracker for initial signal subspace estimation and the solution of the quadratic form which leads to the blind channel estimate is also adaptively computed. It has been discussed and shown by means of computer simulations that although the YAST algorithm might diverge when the iterations reach the dimension of the signal subspace, it can still be used for initial fast convergence followed by other subspace trackers such as OPAST while keeping a global complexity of $O(m n)$. The resulting hybrid method achieves a remarkable convergence speed and numerical stability.

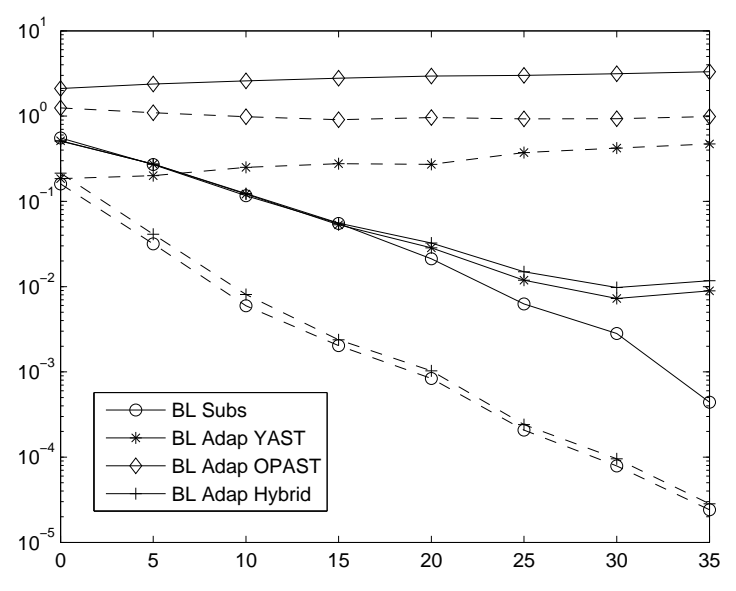

Fig. 2. NMSE versus SNR for different at iterations $k=22$ (solid) and $k=150$ (dashed).

\section{REFERENCES}

[1] J. Cardoso E. Moulines, P. Duhamel and S. Mayrargue. 'Subspace methods for the blind identification of multichannel FIR filters'. IEEE Transactions on Signal Processing, Vol 43(2), pp 516-525, February 1995.

[2] L. Tong and S. Perreau. 'Multichannel Blind Identification: From Subspace to Maximum Likelihood Methods'. Proceedings of IEEE, Vol 86(10), October 1998.

[3] Shengli Zhou, B. Muquet and G.B. Giannakis. 'Subspacebased (semi-) blind channel estimation for block precoded space-time OFDM'. IEEE Transactions on Signal Processing, Vol 50(5), pp. 1215-1228, May 2002.

[4] X.G. Doukopoulos and G.V. Moustakides. 'Adaptive power techniques for blind channel estimation in CDMA systems'. IEEE Transactions on Signal Processing, Vol 53(3), pp. 1110-1120, March 2005.

[5] A. Gorokhov and Ph. Loubaton. 'Semi-blind second order identification of convolutive channels'. Proc. of ICASSP, pp. 3905-3908, 1997.

[6] R. Badeau, B. David and G. Richard. 'Yet Another Subspace Tracker'. Proc. of ICASSP, Vol IV, pp. 329-332, 2005.

[7] C.E. Davila. 'Efficient, high performance, subspace tracking for time-domain data'. IEEE Transactions on Signal Processing, Vol 48(12), pp. 3307-3315, Dec 2000.

[8] D.J. Rabideau. 'Fast, rankadaptive subspace tracking and applications'. IEEE Transactions on Signal Processing, Vol 44(9), pp. 2229-2244, Sept 1996.

[9] D.J. Abed-Meraim, A. Chkeif and Y. Hua. 'Fast orthonormal PAST algorithm'. IEEE Signal Proc. Letters, Vol 7(3), pp. 60-62, Mar 2000.

[10] R. Badeau, B. David and G. Richard. 'Fast approximated power iteration subspace tracking'. IEEE Transactions on Signal Processing, Vol 53(8), pp. 2931-2941, August 2005.

[11] G.H. Golub and C.F. Van Loan. Matrix computations, Johns Hopkins, 3rd edition, 1996. 